\title{
Synergistic Effects of Brain-Derived Neurotrophic Factor and Chondroitinase ABC on Retinal Fiber Sprouting after Denervation of the Superior Colliculus in Adult Rats
}

\author{
Daniela Tropea, ${ }^{\star}$ Matteo Caleo, ${ }^{\star}$ and Lamberto Maffei \\ Istituto di Neuroscienze del Consiglio Nazionale delle Ricerche, and Scuola Normale Superiore, 56100 Pisa, Italy
}

\begin{abstract}
Damage to the adult CNS often causes devastating and permanent deficits because of the limited capacity of the brain for anatomical reorganization. The finding that collateral sprouting of uninjured fiber tracts mediates recovery of function prompts the search for experimental strategies that stimulate axonal plasticity after CNS trauma. Here we characterize treatments that promote the sprouting of undamaged retinal afferents into the denervated superior colliculus (SC) after a partial retinal lesion in the adult rat. Delivery of brainderived neurotrophic factor (BDNF) was performed to enhance the intrinsic potential of retinal ganglion cells to reelongate their axons. Reduction of the neurite growth-inhibitory properties of the adult $\mathrm{SC}$ was accomplished via treatment with chondroitinase $\mathrm{ABC}(\mathrm{C}-\mathrm{ABC})$, which degrades chondroitin sulfate proteoglycans. Retinal axons were labeled via intraocular injections of fluorescently tagged cholera toxin B subunit, and fiber sprouting within the denervated SC was measured by quantitative laser-scanning confocal microscopy 1 week after the retinal lesion. We found that both the administration of BDNF and the injection of $\mathrm{C}-\mathrm{ABC}$ induce significant sprouting of retinal afferents into the collicular scotoma. Remarkably, the combined treatment with BDNF and C-ABC showed synergistic effects on axon growth. Colocalization analysis with anti-synapsin antibodies demonstrated synapse formation by the sprouting axons. These results suggest that the combined treatment with BDNF and C-ABC can be relevant in therapies for the repair of the damaged adult CNS.
\end{abstract}

Key words: CNS injury; brain-derived neurotrophic factor; chondroitinase $\mathrm{ABC}$; proteoglycans; fiber sprouting; synaptic reorganization

\section{Introduction}

Lesions to the adult CNS show the inability of the damaged cells to regenerate their axons and a limited capacity for reorganization of the fiber tracts that have been spared by the injury (Fouad et al., 2001; Chen et al., 2002b). In motor systems, there is ample evidence that stimulating the growth of undamaged axons induces functional recovery even in the absence of regeneration of the severed fibers (Thallmair et al., 1998; Z'Graggen et al., 1998; Raineteau and Schwab, 2001; Weidner et al., 2001; Chen et al., 2002a). These data implicate plasticity of unlesioned fiber tracts as a mechanism for repair and prompt the search for treatments that promote collateral formation after CNS lesions.

Retinal ganglion cells (RGCs) have been extensively used to study the potential of central neurons to undergo plastic rearrangements after traumatic injury. It is known that adult RGCs lack the ability to reinnervate areas of the superior colliculus (SC) that have lost their normal retinal input (Lund et al., 1973; Col-

Received Nov. 1, 2002; revised May 27, 2003; accepted June 2, 2003.

This work was supported by a grant from the International Institute for Research in Paraplegia. Regeneron Pharmaceuticals kindly provided recombinant human BDNF. The anti-phospho-Trk antibody was a kind gift from $\mathrm{F}$ Watson. We are particularly indebted to L. Gianfranceschi and C. Lodovichi for help during the first phases of this study. We thank G. Ratto for participating in the data collection for Fig. 1, A. Viegi for help with Western blot experiments, P. Guasoni for statistical advice, and J. W. Fawcett, A. Cellerino, and T. Pizzorusso for comments on this manuscript. We are also grateful to C. Palla, G. C. Cappagli, C. Orsini, and A. Tacchi for excellent technical assistance. *D.T. and M.C. contributed equally to this study.

Correspondence should be addressed to Dr. Matteo Caleo, Istituto di Neuroscienze del Consiglio Nazionale delle Ricerche, via G. Moruzzi 1, 56100 Pisa, Italy. E-mail: caleo@in.cnr.it.

Copyright $\odot 2003$ Society for Neuroscience $\quad$ 0270-6474/03/237034-11\$15.00/0 onnese and Constantine-Paton, 2001). For example, removal of one eye in the rat causes vigorous sprouting of the axons of the remaining eye into the denervated SC but only when the enucleation is performed within the third postnatal week (Lund et al., 1973; Bastos et al., 1999). This lack of sprouting of the adult retinotectal projection is probably attributable to a complex interplay of factors both intrinsic and extrinsic to the mature RGCs (So and Yip, 1998; Chierzi and Fawcett, 2001). Intrinsic factors include the inability of adult RGCs to activate cellular responses required for axonal elongation. Experimental manipulations that increase the growth potential of adult RGCs allow their axons to overcome external influences and to regenerate across crush lesions of the optic nerve (Berry et al., 1996; Lehmann et al., 1999; Leon et al., 2000). Extrinsic factors are elements of the extracellular environment that counteract potential attempts of the adult RGCs to grow new connections. Multiple inhibitors of neurite growth have been identified in the CNS parenchyma, including Nogo-A, myelin-associated glycoprotein, and chondroitin sulfate proteoglycans (CSPGs; Bandtlow and Zimmermann, 2000; Brittis and Flanagan, 2001; Fournier and Strittmatter, 2001; Domeniconi et al., 2002). In particular, CSPGs are components of the extracellular matrix inhibitory for axonal sprouting and growth. It has been recently shown that degradation of CSPG glycosaminoglycan $(\mathrm{GAG})$ side chains via treatment with the bacterial enzyme chondroitinase $\mathrm{ABC}(\mathrm{C}-\mathrm{ABC})$ promotes $\mathrm{CNS}$ regeneration (Moon et al., 2001; Bradbury et al., 2002).

Here we produced partial retinal lesions in adult rats to iden- 
tify treatments that promote the sprouting of undamaged retinal axons into the deafferented SC. The hypothesis tested in this paper is that axonal sprouting can be powerfully stimulated by acting simultaneously on both intrinsic and extrinsic factors, i.e., by combining experimental interventions that enhance the growth potential of adult RGCs with strategies that change the CNS environment into a more permissive terrain. Delivery of brain-derived neurotrophic factor (BDNF) has been used to enhance the intrinsic ability of RGCs to extend new connections. Reduction of the neurite-growth inhibitory properties of the adult tectum was accomplished by degrading CSPG glycosaminoglycan side chains with $\mathrm{C}-\mathrm{ABC}$.

\section{Materials and Methods}

Animal treatment. A total of 104 young adult Long-Evans rats (250 gm body weight) were used in this study. All experimental procedures were approved by the Italian Ministry of Health. For eye lesions, the animals were anesthetized with avertin and placed in a stereotaxic apparatus. The sclera was exposed with a retro-orbital approach and touched with a heat microcauterizer, resulting in the complete disruption of retinal cells and the optic nerve fiber layer in the heated area. Lesions were made in the inferior-temporal portion of the retina at a distance of $1-2 \mathrm{~mm}$ from the optic nerve (Fig. 1 $A, B$ ). Fifteen animals with partial retinal lesions were left untreated and analyzed at 1 or 3 weeks postoperatively.

Ten rats were implanted with osmotic minipumps (model 1007D; Alzet, Palo Alto, CA) immediately after the retinal scotoma. Minipumps (pumping rate, $0,5 \mu \mathrm{l} / \mathrm{hr}$ ) were connected via polyethylene tubing to a stainless steel cannula ( 30 gauge) implanted into the SC contralateral to the lesioned eye. Osmotic minipumps were filled with recombinant human BDNF (Regeneron Pharmaceuticals, Tarrytown, NY) at a concentration of $4 \mu \mathrm{g} / \mu \mathrm{l}$. Control animals received minipump infusions of cytochrome $c$ (a protein with physicochemical properties similar to those of BDNF; Sigma, St. Louis, MO) at the same concentration. Animals were continuously infused for a period of 1 week.

Eleven rats received intravitreal injections of BDNF (Regeneron; $1 \mu \mathrm{l}$ of a $10 \mu \mathrm{g} / \mu \mathrm{l}$ solution) or cytochrome $c$ into the damaged eye. Injections were made immediately after the retinal damage and then 3 or $4 \mathrm{~d}$ later, and the animals were killed at 1 week. Eye injections were performed under avertin anesthesia with a pulled micropipette connected to a microinjector. The micropipette was inserted at the ora serrata, and the injection volume was slowly released into the vitreous. All injections were made in correspondence with the nasal portion of the eye.

Five rats were treated with collicular microinjections of protease-free C-ABC (Seikagaku, Tokyo, Japan) in concomitance with and $3 \mathrm{~d}$ after the retinal scotoma. Injections of $750 \mathrm{nl}$ of a $48 \mathrm{U} / \mathrm{ml}$ solution of the enzyme were made into the superior colliculus by means of a glass pipette $(50 \mu \mathrm{m}$ tip diameter) mounted on a motorized $(0.1 \mu \mathrm{m}$ step) three-axis micromanipulator and connected to a microinjector (NA-1; Sutter Instruments, Novato, CA). Coordinates with respect to $\lambda$ were $1 \mathrm{~mm}$ lateral, $300 \mu \mathrm{m}$ anterior, and $2.6 \mathrm{~mm}$ deep from the pial surface.

Five rats were simultaneously treated with $\mathrm{C}-\mathrm{ABC}$ and with eye injections of BDNF using the procedures described above.

To visualize retinal ganglion cell axons at the collicular level, injections of Alexa 594-conjugated subunit B of cholera toxin (CTB; Molecular Probes, Leiden, The Netherlands) were administered in the lesioned eye. A total of $3 \mu \mathrm{l}$ of $1 \%$ CTB in water was administered into the eye $2 \mathrm{~d}$ before tissue processing.

Immunohistochemistry. All rats were perfused with $4 \%$ paraformaldehyde in $0.1 \mathrm{~m}$ phosphate buffer. The brains were dissected and placed in $30 \%$ sucrose in PBS. Coronal sections $40 \mu \mathrm{m}$ thick were cut on a freezing microtome and collected in PBS.

To check the diffusion of BDNF after minipump infusion into the SC, a rabbit anti-BDNF antibody (Chemicon, Temecula, CA) was used. Sections were treated with $0.01 \%$ Triton X-100 and $10 \%$ normal goat serum in PBS for $1 \mathrm{hr}$ at room temperature. Incubation with the primary antibody at a concentration of $2.5 \mu \mathrm{g} / \mathrm{ml}$ was performed for $48 \mathrm{hr}$ at $4^{\circ} \mathrm{C}$. Sections were then treated with a secondary antibody $(7.5 \mu \mathrm{g} / \mathrm{ml}$ biotinylated goat anti-rabbit; Vector Laboratories, Burlingame, CA) for $3 \mathrm{hr}$ at room temperature and with an avidin-biotin-peroxidase complex (1: 100 dilution; ABC Elite kit; Vector Laboratories) for $1 \mathrm{hr}$ at room temperature, followed by a nickel-enhanced diaminobenzidine (DAB) reaction.

To test the effectiveness of chondroitinase $\mathrm{ABC}$, we used the antibody 2B6 (Seikagaku), which binds to a four-sugar stub that is left behind on CSPGs after chondroitinase digestion (Moon et al., 2001). Slices were preincubated in Tris-buffered saline (TBS) containing $1 \%$ goat serum for $1 \mathrm{hr}$ at room temperature and then incubated with the primary biotinylated antibody $(20 \mu \mathrm{g} / \mathrm{ml})$ overnight at $4^{\circ} \mathrm{C}$. After washing, sections were incubated with an avidin-biotin-peroxidase complex (1:100 dilution; ABC Elite kit; Vector Laboratories) for $1 \mathrm{hr}$ at room temperature and then subjected to the $\mathrm{DAB}$ reaction.

Synapsin immunodetection was performed with the mouse monoclonal antibody 355 (Chemicon) at a concentration of $2 \mu \mathrm{g} / \mathrm{ml}$. Serial sections were preincubated in a mix containing $10 \%$ horse serum (Sigma) and $0.3 \%$ Triton X-100 in PBS for $1 \mathrm{hr}$ at room temperature. They were then reacted overnight at $4^{\circ} \mathrm{C}$ with the primary antibody diluted in PBS containing $0.3 \%$ Triton X-100 and $1 \%$ serum. The following day, the sections were rinsed in PBS and then incubated with biotinylated antimouse antibody (1:200; Vector Laboratories) for $3 \mathrm{hr}$ at room temperature. After washing, sections were incubated with fluorescein-conjugated avidin (Vector Laboratories) and then mounted with antifading agent (Vectashield; Vector Laboratories).

For detecting microglial reactivity, slices were preincubated in $10 \%$ horse serum and $0.2 \%$ Triton X-100 in PBS and then reacted overnight in the presence of the OX-42 antibody (PharMingen, San Diego, CA; mouse monoclonal, 1:500) in blocking buffer. On the following day, the sections were incubated with a biotinylated secondary antibody followed by the $\mathrm{ABC}$ kit and the $\mathrm{DAB}$ reaction.

In a subset of animals devoted to measuring colocalization of synapsin I-positive puncta with retinal axons, $4 \mu \mathrm{l}$ of unlabeled CTB ( $1 \%$ solution; Calbiochem, San Diego, CA) was injected intravitreally 2 d before killing. Double-label immunofluorescence for CTB and synapsin I was performed as follows. Slices were postfixed in $4 \%$ paraformaldehyde for $4 \mathrm{hr}$, washed in PBS, and incubated in a glycine solution $(0.1 \mathrm{M})$ for $30 \mathrm{~min}$. Sections were then preincubated overnight at $4^{\circ} \mathrm{C}$ in the following mix (in \%): 5 normal rabbit serum (NRS), $2.5 \mathrm{BSA}$, and 0.5 Triton X-100 in PBS. The day after, they were rapidly rinsed in PBS and incubated in the primary antibody [1:4000 goat anti-CTB; Calbiochem; incubation mix (in \%): 2 NRS, $2.5 \mathrm{BSA}$, and 2 Triton $\mathrm{X}-100$ in PBS) for $3 \mathrm{~d}$ at $4^{\circ} \mathrm{C}$. After washes, slices were incubated with Alexa 488-conjugated rabbit anti-goat antibody (Molecular Probes). Synapsin immunolabeling was then performed as described above. Bound anti-synapsin antibodies were revealed with biotinylated secondary antibodies followed by Сy3conjugated extravidin (Sigma).

Measurement of the extent of the retinal damage and dimensions of the collicular scotoma. After perfusion of the animals, the lesioned eye was dissected out with a small stump of optic nerve attached. Small crystals of the lipophilic tracer DiI (Molecular Probes) were applied on the stump of the nerve, and the entire eye was then placed in fixative (4\% paraformaldehyde in $0.1 \mathrm{M}$ phosphate buffer) for at least 4 weeks at $37^{\circ} \mathrm{C}$ to allow retrograde filling of retinal ganglion cells (see Fig. $1 A$ ). The retina was flat-mounted on a slide and examined with epifluorescence. Both the total area of the retina and the area of the retina occupied by the lesion were determined with Neurolucida (Microbrightfield, Colchester, VT; see Fig. $1 B)$.

For recostruction of the collicular scotoma, sections through the SC were kept in serial order, and the CTB label was examined with a fluorescence microscope equipped with Neurolucida (Microbrightfield). One in three sections was used for the analysis. In each section, the superficial gray was drawn at $10 \times$ magnification, and its area was measured. The outline of the scotoma was also drawn. The total volume of the superficial gray as well as the volume of the scotoma were estimated (Fig. $1 C, D)$. For each animal, the fraction of the SC occupied by the scotoma was then calculated.

Analysis of fiber and synaptic density within the scotoma. Both fiber density and synaptic density analysis were performed on serial sections (one of three). All confocal images were collected with an Olympus Op- 
tical (Tokyo, Japan) confocal microscope using a $63 \times$ water immersion lens with numerical aperture 0.9. Settings for laser intensity, gain, offset, and pinhole size were optimized initially and held constant through the study. During image collection, confocal settings were regulated so that the full range of pixel intensities $(0-255)$ was used, with very little saturation at either end of the intensity range. Examination of animals from the various treatment groups was interdigitated to avoid bias caused by slow shifts in laser power. For each coronal section containing the scotoma, representative fields $(120 \times 80 \mu \mathrm{m})$ were acquired using the same settings in the undeprived portion of the SC, in a border region (centered at a distance of $100 \mu \mathrm{m}$ from the boundary of the scotoma), and in the center of the scotoma (see Fig. 1C). On average, center fields were positioned $380 \pm 76 \mu \mathrm{m}$ (mean $\pm \mathrm{SD})$ from the edge of the scotoma. All fields were located in the stratum griseum superficiale. For analysis of CTB-labeled fibers, each confocal image consisted of the projection of five confocal sections taken at $3 \mu \mathrm{m}$ intervals. Because penetration of anti-synapsin antibody within the tissue was limited (Silver and Stryker, 2000a; Smith et al., 2000), stacks of images were collected at the top face of the tissue section, and the image within each stack with the highest average pixel intensity was selected for the quantitative analysis of synapsin immunoreactivity.

All image analysis was performed using MCID/M4 software (Imaging Research, St. Catharines, Ontario, Canada). The density of sprouting into the scotoma was calculated for each confocal micrograph by creating a binary image with a threshold value chosen to include all in-focus axons and arbors and to exclude background fluorescence and calculating the percentage of positive pixels in the field. To compensate for tracing efficiency in individual animals, the percentage of positive pixels within the scotoma was normalized to axon density outside the scotoma. Therefore, for each coronal section, normalized values of fiber density were obtained at the edge and in the center of the scotoma, and normalized data obtained in different sections were averaged to obtain the value for a particular animal.

The area occupied by synapsin-positive pixels was calculated for each image by applying a threshold that distinguishes background levels of fluorescence from the intense punctate staining characteristic of synapsin immunoreactivity (Silver and Stryker, 2000a; Smith et al., 2000). Calculation of the cross-sectional area of synapsin staining was used here and in previous studies (Smith et al., 2000) to estimate the density of presynaptic boutons. All image analysis was performed blind to the experimental treatment. To compensate for possible differences in the quality of the immunostaining from animal to animal and section to section, the field corresponding to the intact SC served as a within-section reference. All synaptic density data obtained within the scotoma were therefore normalized to the values derived from the adjacent undeafferented SC.

Determination of the density profile of the CTB label across the scotoma. Coronal sections through the SC of three control lesioned and five BDNF- and C-ABC-treated rats were used for the analysis. Confocal images comprising the whole extent of the collicular scotoma were acquired with a $20 \times$ objective and fed to an image analyzer (MCID/M4; Imaging Research). An open-circle cursor whose diameter could be adjusted to the width of the superficial gray was used to scan density values from the edge of the scotoma to midline. To control for variations in background levels of fluorescence across sections, the density readings within the scotoma were subtracted by the average of density readings from the contralateral unlabeled superficial gray of each coronal section. Density profiles corrected for background density were averaged to obtain the profile in a single rat (3-10 coronal sections per animal). Curves from single animals were then averaged to obtain the profile for each experimental group.

Colocalization analysis. Colocalization of CTB-labeled retinal axons with synapsin I-positive clusters was performed at the border and in the center of the scotoma using the methods and software developed by Dr. M. Silver (University of California, San Francisco, CA) (Silver and Stryker, 2000a). Two stacks of optical sections separated by $1 \mu \mathrm{m}$ were collected on a confocal microscope (Olympus). One stack contained images of the synapsin I label, whereas the other contained images of the labeled axons. All images were collected through a $60 \times$ objective. Image size was $800 \times 600$ pixels, resulting in a pixel size of $0.133 \mu \mathrm{m}$. One optical section in the synapsin I stack was selected for colocalization analysis and called the reference slice. All pixels of the reference sections located within cell bodies or blood vessels were masked and removed from the analysis. The image was then thresholded so that only the brightest $10 \%$ of the neuropil pixels were retained, and all others were set to zero. This procedure effectively separates most of the presynaptic sites from one other in the $x-y$ plane of the reference section (Silver and Stryker, 2000a). The location of these synaptic puncta along the $z$-axis was determined by comparing pixel intensity values in the reference slice with corresponding pixel intensities in the slices immediately above and immediately below the reference section. We then manually traced the portions of each CTB-labeled axon that were located within the focal plane of interest (corresponding to the reference slice in the synapsin I stack). Only those presynaptic puncta that were located entirely within a traced axon segment in all three dimensions were considered colocalized. To estimate the amount of colocalization expected on the basis of random overlap of the two labels, we performed the same colocalization analysis on a synapsin I stack and an axon stack taken from different fields ("shuffled" condition; Silver and Stryker, 2000a). The density of colocalized synapses (i.e., the number of colocalized presynaptic clusters per unit area of traced axon) in the shuffled condition was often equal to zero and consistently lower than that obtained from stacks of images taken from the same location in the tissue section.

Immunoblotting. Immunoblotting was performed essentially as described by Viegi et al. (2002). Proteins from superior colliculi of C-ABCinjected and control animals were extracted with modified radioimmunoprecipitation assay buffer ( $1 \%$ Triton X-100, 0.5\% Na deoxycholate, $0.1 \%$ SDS, $10 \%$ glycerol, $20 \mathrm{~mm}$ Tris- $\mathrm{HCl}, \mathrm{pH} 8,150 \mathrm{~mm} \mathrm{NaCl}, 1 \mathrm{~mm}$ EDTA, $50 \mathrm{~mm} \mathrm{NaF}, 1 \mathrm{~mm} \mathrm{Na}_{2} \mathrm{MoO}_{4}, 0.5 \mathrm{~mm} \mathrm{Na}_{3} \mathrm{VO}_{4}, 5 \mathrm{~mm} \mathrm{Na}_{4} \mathrm{P}_{2} \mathrm{O}_{7}, 10$ $\mu \mathrm{g} / \mathrm{ml}$ leupeptin, $10 \mu \mathrm{g} / \mathrm{ml}$ aprotinin, and $0.01 \mathrm{~mm}$ PMSF), and the total concentration of the samples was assessed with a protein assay kit (BioRad, Hercules, CA) using a bovine serum albumin-based standard curve. The same amount of total protein from treated and control animals (a total of $100 \mu \mathrm{g}$ ) was electrophoresed with $10 \%$ SDS-PAGE. Proteins were transferred to nitrocellulose overnight at $4^{\circ} \mathrm{C}$. Blots were blocked with $5 \%$ dry milk powder and $0.05 \%$ Tween 20 in TBS for $2 \mathrm{hr}$ and incubated with anti-phospho-Trk antibody (a gift from Dr. F. Watson, Harvard Medical School, Boston, MA) in TBS containing 0.05\% Tween 20 and 2\% milk powder overnight at $4^{\circ} \mathrm{C}$. After washing, blots were incubated for 2 hr with horseradish peroxidase-conjugated secondary antibody (goat anti-rabbit, $0.3 \mu \mathrm{g} / \mathrm{ml}$ ), developed by means of an enhanced chemiluminescence system (Amersham Biosciences, Uppsala, Sweden) and captured on autoradiographic films (Amersham Biosciences Hyper ECL). After development for anti-phospho-Trk, blots were stripped with a ReBlot recycling kit (Chemicon) and reprobed with anti-pan Trk antibody ( $1 \mu \mathrm{g} / \mathrm{ml}$; Santa Cruz Biotechnology, Santa Cruz, CA).

For densitometric analysis, films were digitalized with a camera, and band ODs were measured with MCID/M4 software. The state of phosphorylation of Trk receptors was evaluated by measuring the OD of the phosphotyrosine band at $140 \mathrm{kDa}$ and dividing it by the OD of the pan-Trk band measured on the same filter after the stripping procedure. The data reported represent the percentage of phosphorylated Trk versus the total amount of Trk present in the same sample.

Statistical analysis. Values of fiber density and synaptic density were analyzed with one-way ANOVA in which each treatment was compared with its specific control and with the untreated lesioned group. The only exception was the combined treatment with BDNF and C-ABC, for which a $t$ test was performed with respect to the untreated control group. Differences between groups were considered significant at $p<0.05$.

For analysis of the synergism between BDNF and C-ABC on fiber sprouting, we tested the hypothesis that the effects of the combined treatment are greater than the sum of the effects of each treatment alone. The effects of BDNF and C-ABC alone were calculated by subtracting the mean value of fiber density in control untreated animals from the fiber density value obtained in each individual animal belonging to either the $\mathrm{BDNF}$ or the $\mathrm{C}-\mathrm{ABC}$ group. The effects of BDNF and $\mathrm{C}-\mathrm{ABC}$ were summed by taking into account the different variances and sizes of each population. 


\section{Results}

The main aim of this study is to identify treatments that allow filling in of the deafferented SC after a partial lesion to the adult rat retina. Strategies that have been tested include delivery of $\mathrm{BDNF}$ and degradation of chondroitin sulfate proteoglycans via treatment with $\mathrm{C}-\mathrm{ABC}$.

Definition of the collicular scotoma after partial retinal lesion We performed focal retinal lesions in adult rats that result in the axotomy of the RGCs located in the ventrotemporal quadrant of the retina. The extent of the retinal damage was visualized by retrograde filling of RGC fibers with DiI (Fig. $1 A, B$ ). For each animal we calculated the fraction of retinal area containing axotomized RGCs. In control lesioned animals at 1 week, the percentage of such area ranged from 6.1 to $14.5 \%$ (8.9 $\pm 1.1 \%$, mean $\pm \mathrm{SE}$ ). The same analysis was performed on the other experimental groups and revealed no significant differences versus controls (one way ANOVA, $p=0.46$ ). This rules out that the differences in the sprouting response after the various manipulations can be ascribed to a systematic bias in the extent of the retinal damage.

Retinal lesions produce a retinotopically appropriate zone of deafferentation (a scotoma) in the contralateral lateral geniculate nucleus and SC. We focused our study on the sprouting of retinal terminals within the scotoma in the superficial gray of the contralateral SC. Retinal ganglion cell axons in the SC were visualized with anterograde transport of fluorophore-conjugated CTB. A single section of SC containing the collicular scotoma and a three-dimensional reconstruction of the scotoma are reported in Figure 1, $C$ and $D$, respectively. In all coronal sections used for the analysis, the boundary of the scotoma was sharp and clearly identifiable, as shown in the representative example of Figure $1 C$.

Our quantitative analysis of fiber sprouting included three fields for each coronal section containing the collicular scotoma (Fig. 1C, boxes): a region in the intact SC, a border region centered at $100 \mu \mathrm{m}$ from the edge of the CTB staining, and a center region (see Materials and Methods). The same fields were used for the evaluation of synaptic density after synapsin immunolabeling.

We have made sure that we have compared fields taken at correspondent positions within the scotoma in control and treated animals on the basis of the following observations. First, we checked that the overall size of the scotoma was not affected by any treatment. Second, we have controlled that the mean dimensions of the scotoma in the single coronal sections were superimposable among the various groups. To address the first issue, in each animal we measured the volume of the scotoma and the total volume of the superficial gray using Neurolucida (Microbrightfield) and calculated the fraction of the SC that was deafferented by the lesion. We found that the scotoma occupied $5.2-15.5 \%$ of the total SC volume in control untreated animals $(8.8 \pm 0.8 \%$, mean \pm SE), and the percentage of the SC deprived of the retinal input did not vary between the experimental groups (one way ANOVA, $p=0.3$ ). To further exclude that biases in the placement of the sampling fields may arise as a consequence of differences in the three-dimensional shape of the scotoma, we measured the lateral extent of the deafferented area in all of the coronal sections used for the quantitative analysis. In each section we measured the distance between midline and the lateral boundary of the scotoma using Neurolucida. We found that the mean dimensions of the scotoma in the single coronal sections did not differ between the various groups (one way ANOVA, $p=0.27$ ).
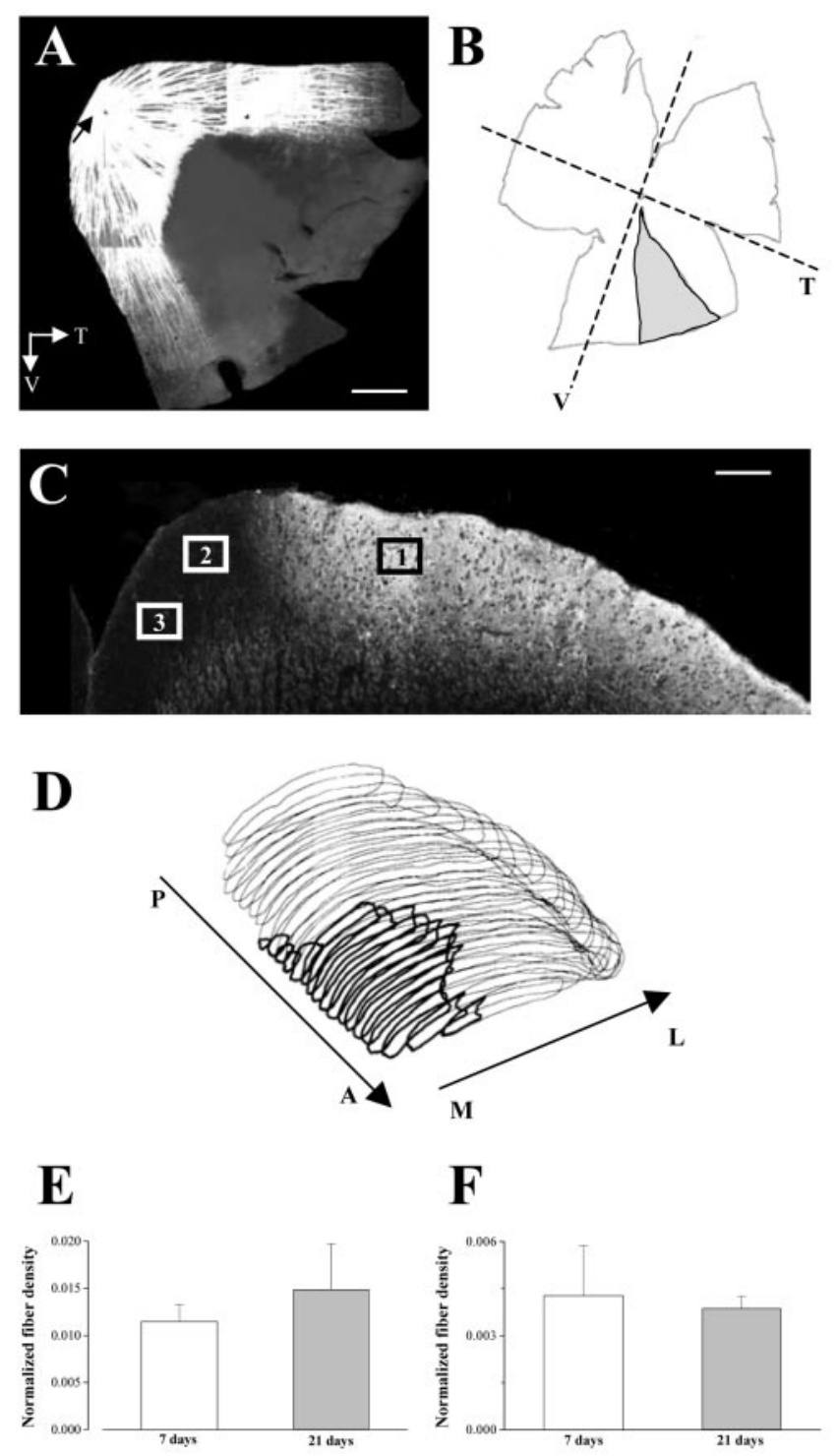

Figure 1. A, Representative example of the ventrotemporal quadrant of a whole-mount lesioned retina in which retinal fibers were retrogradely labeled with Dil. The portion of the retina containing axotomized RGCs is clearly visible (bottom right). An arrow points to the optic disk. V, Ventral; T, temporal. Scale bar, $750 \mu \mathrm{m}$. $B$, Neurolucida drawing of a flat-mount lesioned retina. The extent of the retinal damage is shown in gray. V, Ventral; T, temporal. C, Confocal image of a coronal section through the colliculus of a lesioned animal that was intravitreally injected with Alexa 594-conjugated CTB. The collicular scotoma is visible as the unstained area on the medial part of the superficial gray (left). The three boxes indicate the locations of the fields used for the quantitative analysis: region 1 within the undeprived SC and regions 2 and 3 at the border and in the center of the scotoma, respectively. Scale bar, $200 \mu \mathrm{m}$. D, Tridimensional reconstruction of the right SC (thin lines) containing the collicular scotoma (thick lines). P, Posterior; A, anterior; $M$, medial; $L$, lateral. $E, F$, Retinal fiber density at 7 and $21 \mathrm{~d}$ after the lesion. Neither at the border $(E)$ nor in the center of the scotoma $(F)$ are there significant differences between the two time points. Error bars indicate SE. For each bar, $n=6-8$ rats.

\section{Analysis of fiber sprouting}

We first evaluated fiber sprouting into the collicular scotoma of untreated animals to check whether some axonal plasticity occurs spontaneously over time. Measurement of the density of RGC axon terminals in animals processed 7 and $21 \mathrm{~d}$ after retinal lesion revealed no significant differences either at the border or in the center of the collicular scotoma (Fig. $1 E, F$; $t$ test, $p=0.47 ; p=$ 0.41 , respectively). Thus, no compensatory changes develop spontaneously between 1 and 3 weeks postoperatively. Because 

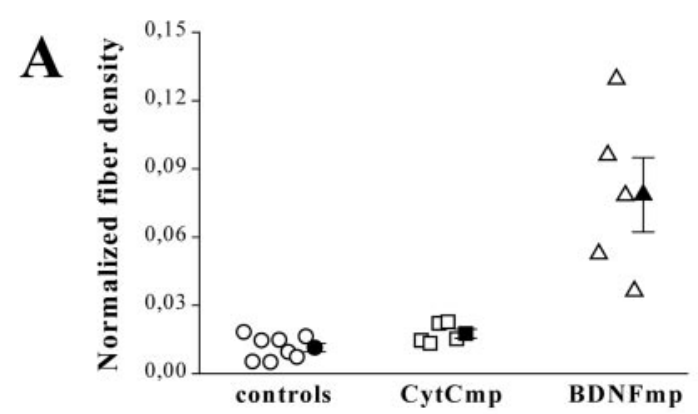

B

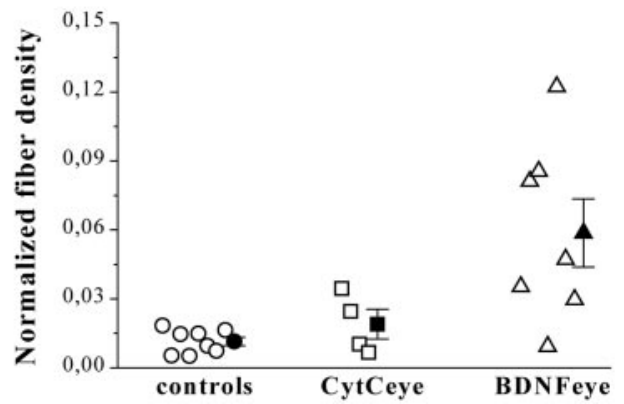

Figure 2. Fiber density values at the border of the scotoma in BDNF-treated animals and corresponding controls. $A$, Effects of BDNF infused into the SC through osmotic minipumps (BDNFmp) compared with those of cytochrome c released in the same way (CytCmp). Controls are untreated lesioned animals. $B$, Effects of BDNF delivered into the lesioned eye (BDNFeye) compared with those obtained in cytochrome c-injected animals (CytCeye). Controls are untreated lesioned rats. Each open symbol represents the value obtained in one animal. The solid symbols indicate the mean for each experimental group. Error bars indicate SE.

exogenous BDNF rapidly modulates axon arborization of the responsive neurons (Cohen-Cory and Fraser, 1995; Alsina et al., 2001), we evaluated the effects of BDNF treatment on retinal fiber sprouting at 1 week.

We first infused BDNF directly at the collicular level by osmotic minipumps. The diffusion of BDNF was checked by performing an immunohistochemical reaction on serial sections containing the SC. For analysis of fiber sprouting, were selected only those slices that were immunolabeled for exogenous BDNF and that were at least $350 \mu \mathrm{m}$ farther from the damage caused by the infusion cannula. Control animals were implanted with minipumps containing cytochrome $c$ at the same concentration as BDNF $(4 \mu \mathrm{g} / \mu \mathrm{l})$. The results show that BDNF delivery was remarkably effective in promoting fiber sprouting at the border of the scotoma in all infused animals (Fig. 2A). Indeed, the statistical analysis indicates that fiber density in the BDNF-treated animals was significantly greater than that in both untreated and cytochrome $c$-infused rats (one way Kruskal-Wallis ANOVA, $p=0.003$; post hoc Dunn's test, $p<0.05$ ). In contrast, values in the latter groups did not differ from each other (ANOVA; post hoc Dunn's test, $p>0.05)$. Analysis of fiber density in the center of the scotoma, however, revealed no significant effects of the BDNF infusion (one-way Kruskal-Wallis ANOVA, $p=0.11$; data not shown).

In a second experimental group, $\mathrm{BDNF}(10 \mu \mathrm{g})$ was injected into the damaged eye at the time of the lesion and then 3-4 d later. To check for possible aspecific effects of the injection procedure, four animals received intravitreal administrations of cytochrome $c$ with the same time schedule. At the border of the scotoma, intraocular BDNF produced a significant increase in fiber density with respect to both control groups (one-way Kruskal-Wallis ANOVA, $p<0.05$; post hoc Dunn's test, $p<0.05$;
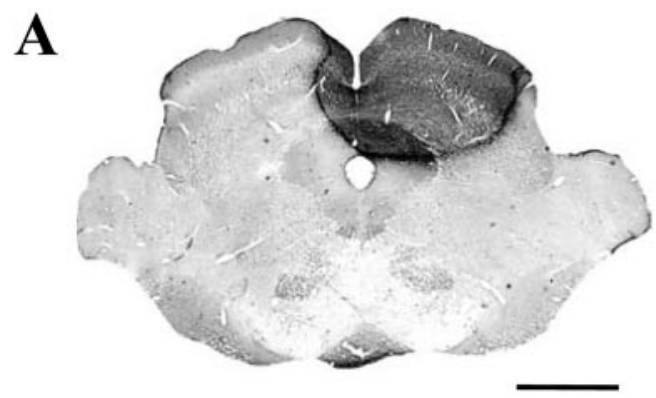

B
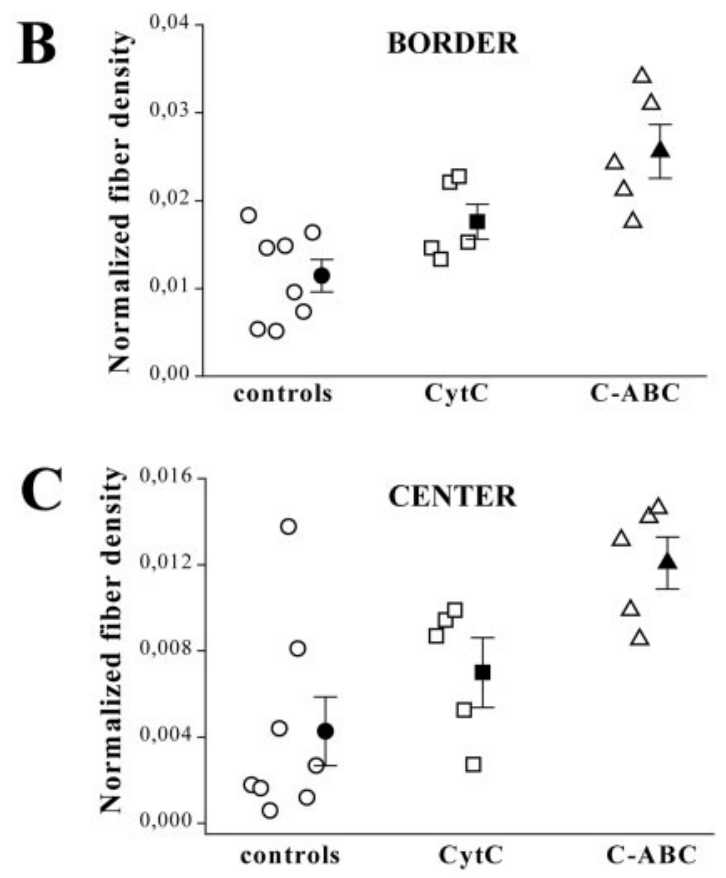

Figure 3. $A$, Coronal section through the midbrain of an animal that was injected into the $S C$ with C-ABC. 2B6 staining shows CSPG degradation in the SC treated with C-ABC. Scale bar, 1.5 $\mathrm{mm} . B, C$, Effects of C-ABC treatment on RGC fiber density at the border $(B)$ and in the center of the collicular scotoma ( $C$. Data in the $C-A B C$ group are compared with those obtained in animals infused with cytochrome c and in untreated lesioned rats. Each open symbol represents the value obtained in one animal. The solid symbols indicate the mean for each experimental group. Error bars indicate SE.

Fig. $2 B$ ). In the same animals, no differences in fiber density were detected in the center of the scotoma (one-way Kruskal-Wallis ANOVA, $p>0.05$; data not shown).

Because CSPGs are potent inhibitors of neurite growth, in an additional group of animals we tested the effects of the treatment with $\mathrm{C}-\mathrm{ABC}$, which prunes the glycosaminoglycan chains of CSPGs. We stereotaxically injected C-ABC into the SC at the time of the retinal lesion. A second injection of $\mathrm{C}-\mathrm{ABC}$ was made $3 \mathrm{~d}$ later, and the anatomical analysis was performed at $7 \mathrm{~d}$. Degradation of CSPG glycosaminoglycan side chains within the injected SC was confirmed using immunostaining with the antibody 2B6, which selectively labels digested CSPGs (Fig. 3A) (Moon et al., 2001; Bradbury et al., 2002). Results of the chondroitinase treatment were compared with those obtained in untreated rats and in rats infused with cytochrome $c$. We found that degradation of CSPGs promoted retinal fiber sprouting not only at the border but also in the center of the collicular scotoma (Fig. $3 B, C$, respectively). At the border, fiber density in the C-ABCtreated group was significantly higher than in both control groups (one-way ANOVA, $p=0.006$; post hoc Tukey test, $p<$ $0.05)$. In contrast, animals infused with cytochrome $c$ and un- 

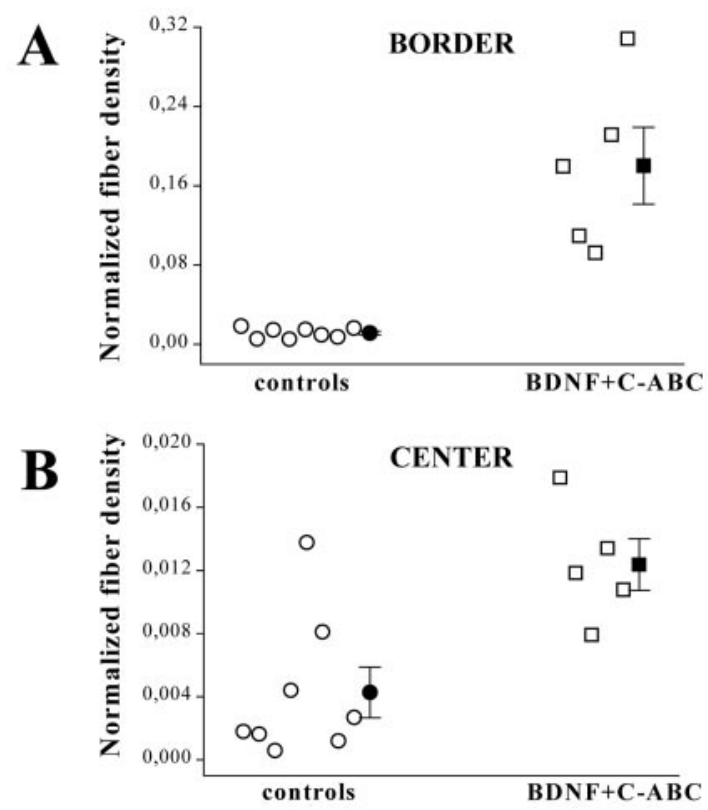

Figure 4. Effects of the combined delivery of BDNF and C-ABC on RGC fiber density at the border $(A)$ and in the center of the collicular scotoma $(B)$. Controls are lesioned animals without any treatment. Each open symbol represents the value obtained in one animal. The solid symbols indicate the mean for each experimental group. Error bars indicate SE.

treated animals did not differ from each other (post-ANOVA Tukey test, $p>0.05)$. ANOVA demonstrated significant effects of C-ABC treatment also in the center of the scotoma (ANOVA, $p=0.01$; post hoc Tukey test, rats in the C-ABC group differ from both control lesions, $p<0.05$ ). Here, the chondroitinase treatment doubled on average the density of RGC fibers with respect to controls (Fig. 3C).

Because both BDNF and C-ABC significantly increase fiber density within the collicular scotoma, we checked the effects of combining the two treatments. In a separate group of animals we performed BDNF eye injection and stereotaxic delivery of C-ABC into the $\mathrm{SC}$ at the time of the retinal lesion and then $3 \mathrm{~d}$ later. The rats were killed at 1 week. This experimental protocol was extremely effective in promoting fiber sprouting at the border of the collicular scotoma, as shown in Figure $4 A$ (Mann-Whitney rank sum test, BDNF plus C-ABC rats vs untreated lesioned animals, $p=0.002)$. The combined treatment significantly increased fiber density levels also in the center of the collicular scotoma ( $t$ test, $\mathrm{BDNF}$ plus $\mathrm{C}-\mathrm{ABC}$ rats vs untreated controls, $p=0.006$; Fig. $4 B$ ).

A summary of the effects of the different manipulations on fiber density at the border of the scotoma is reported in Figure 5. The results show that all tested treatments promote sprouting of RGC axon terminals but with different efficacy. BDNF produces undistinguishable results when administered into the tectum or at the RGC bodies ( $t$ test, BDNF minipump vs BDNF eye, $p=$ 0.39 ). C-ABC treatment increases fiber density by more than twofold (Fig. 5). Remarkably, RGC fiber sprouting in the animals with combined delivery of BDNF and C-ABC is higher than the sum of the effects of each treatment alone, indicating a synergistic action of the two manipulations ( $t$ test, effect of BDNF plus $\mathrm{C}-\mathrm{ABC}$ vs sum of the effects of BDNF and C-ABC, $p=0.003$; Fig. $5)$.

Our data also demonstrate that $\mathrm{C}-\mathrm{ABC}$ is the only treatment able to increase RGC axon density in the center of the collicular scotoma. We found indeed no significant differences in the center between $\mathrm{C}-\mathrm{ABC}$ and the combination of BDNF and $\mathrm{C}-\mathrm{ABC}(t$
A

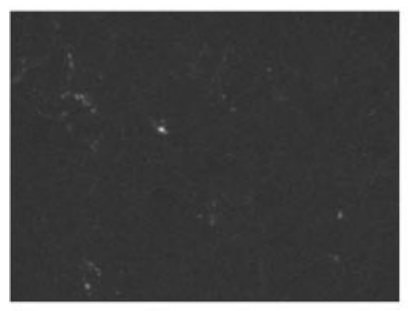

C

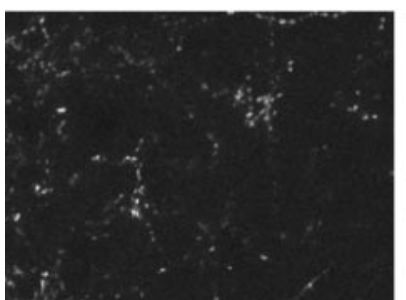

D
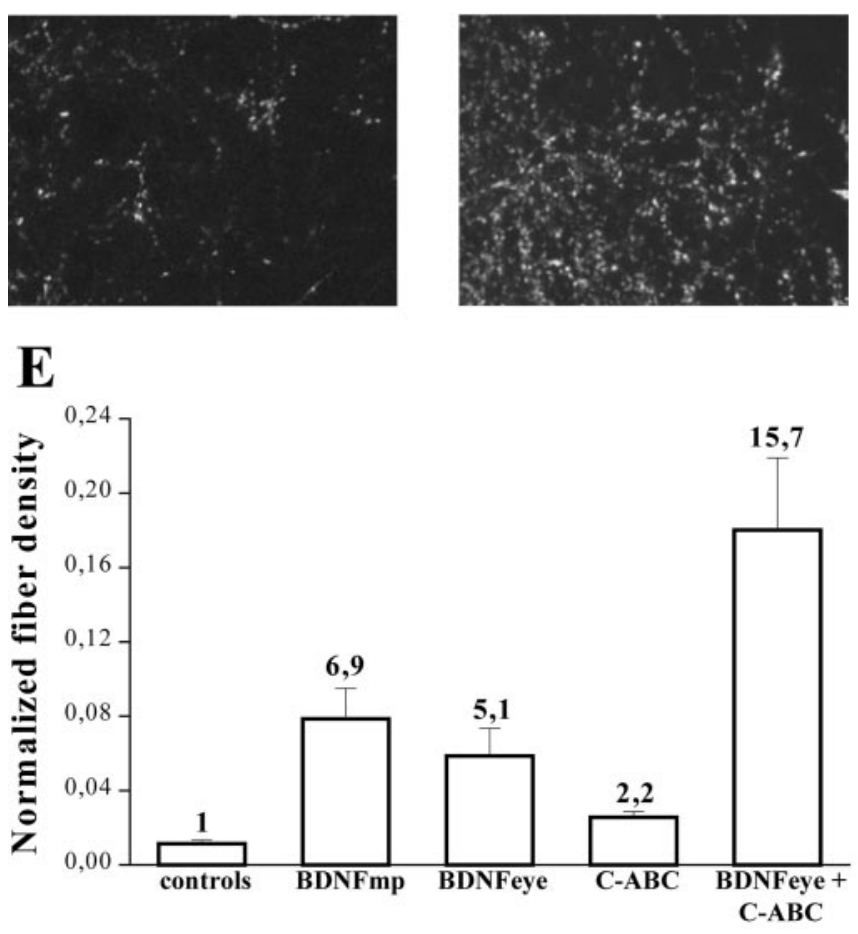

Figure 5. Confocal images of representative fields showing the density of RGC fibers at the border of the scotoma in control and treated rats. Retinal fibers are labeled with Alexa 594conjugated CTB. Untreated animals are shown in $A$. The effect of $C-A B C$ is shown in $B$, intraocular $B D N F$ in $C$, and the combined treatment with BDNF and $C-A B C$ in $D$. Note that fiber sprouting is maximally promoted by BDNF plus $C-A B C$. Brightness and contrast have not been altered in these images; they represent the raw data used to make fiber density measurements. Scale bar, $25 \mu \mathrm{m}$. $E$, Mean values of RGC fiber density observed in the different experimental groups. Numbers on the columns represent the fold increase in fiber density with respect to the mean value observed in untreated lesioned animals at 1 week. Error bars indicate SE. For each histogram, $n=5-8$ rats.

test, $p=0.89$ ), indicating that BDNF does not improve the effects of C-ABC on long-range fiber sprouting. Thus, CSPGs are critically involved in blocking growth of RGC axon collaterals toward the center of the scotoma, and neurotrophin stimulation provides no additive effects.

\section{Spatial distribution of the sprouting within the scotoma of} BDNF- and C-ABC-treated rats

To define more completely the spatial distribution of the sprouting in animals infused with BDNF plus C-ABC, we took confocal images comprising the whole extent of the collicular scotoma (Fig. 6). The density profile of the CTB label inside the scotoma was assessed quantitatively by an image analyzer. This analysis revealed that there is an exponential decay in the density of sprouts starting from the scotoma edge in control lesioned ani- 


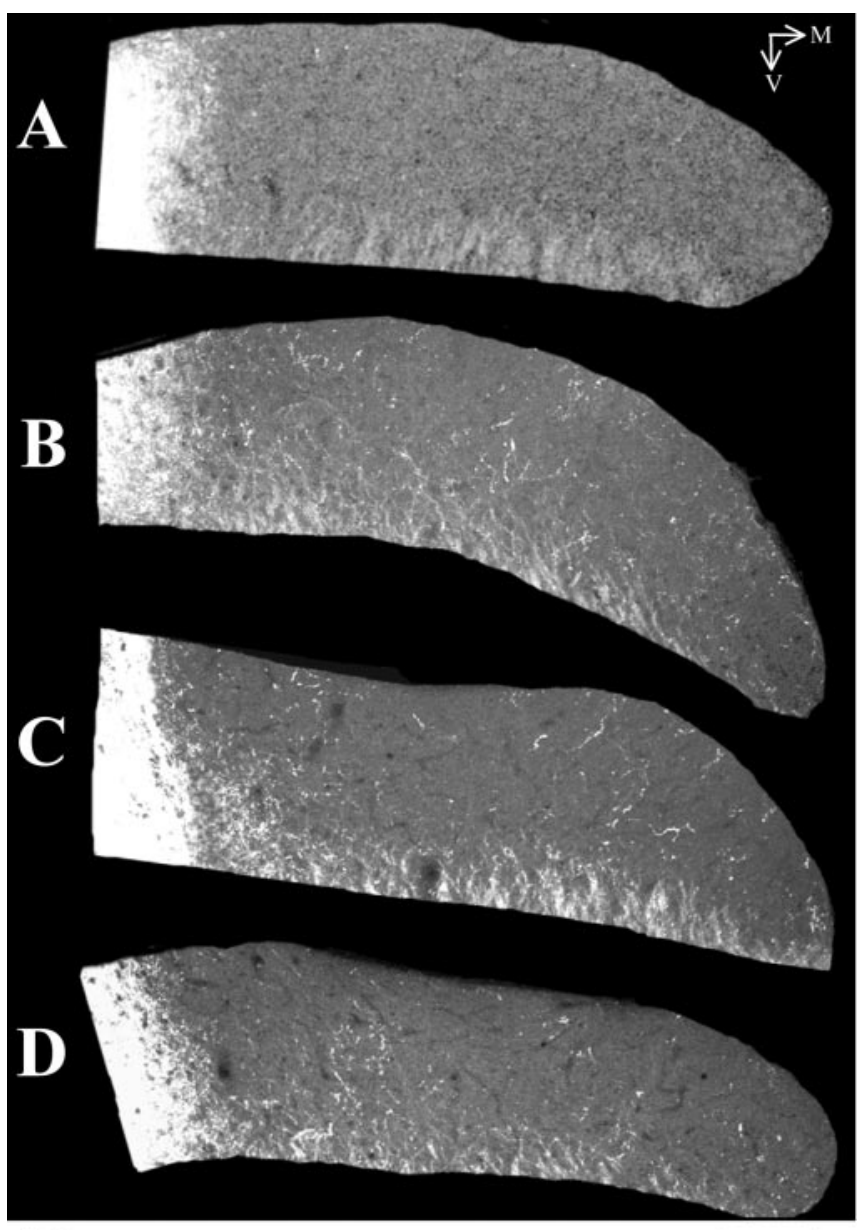

$\mathbf{E}$

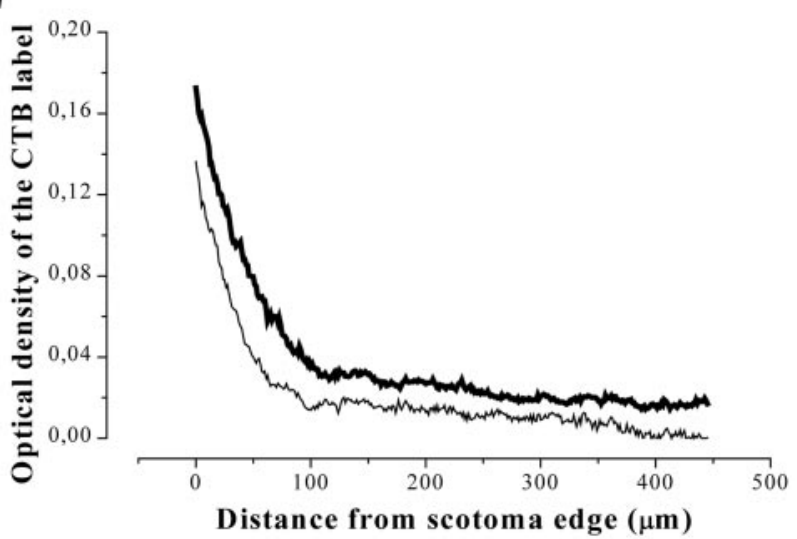

Figure 6. $A-D$, Representative low-power confocal images of the scotoma in control animals $(A)$ and BDNF- and $C-A B C$-infused animals $(B-D)$. The edge of the scotoma is on the left. Many labeled retinal sprouts are visible throughout the superficial gray of all samples from BDNF- and C-ABC-treated animals. M, Medial; V, ventral. Scale bar, $100 \mu \mathrm{m}$. E, Distribution of the density of the (TB label throughout the scotoma in control rats (thin line) and BDNF- and C-ABC-treated rats (thick line). The curves are obtained by averaging data from three control and five BDNF- and C-ABC-treated animals. Fiber quantitation was performed on confocal images taken at relatively low magnification $(20 \times)$, such as those shown in $A-D$. This analysis demonstrates a consistent sprouting response in BDNF- and C-ABC-treated animals with respect to controls.

mals (Fig. 6E). Animals receiving BDNF plus $\mathrm{C}-\mathrm{ABC}$ showed a shallower decay in the density of labeling, indicative of increased sprouting at the border. Moreover, density values in treated animals were consistently higher than those measured in controls
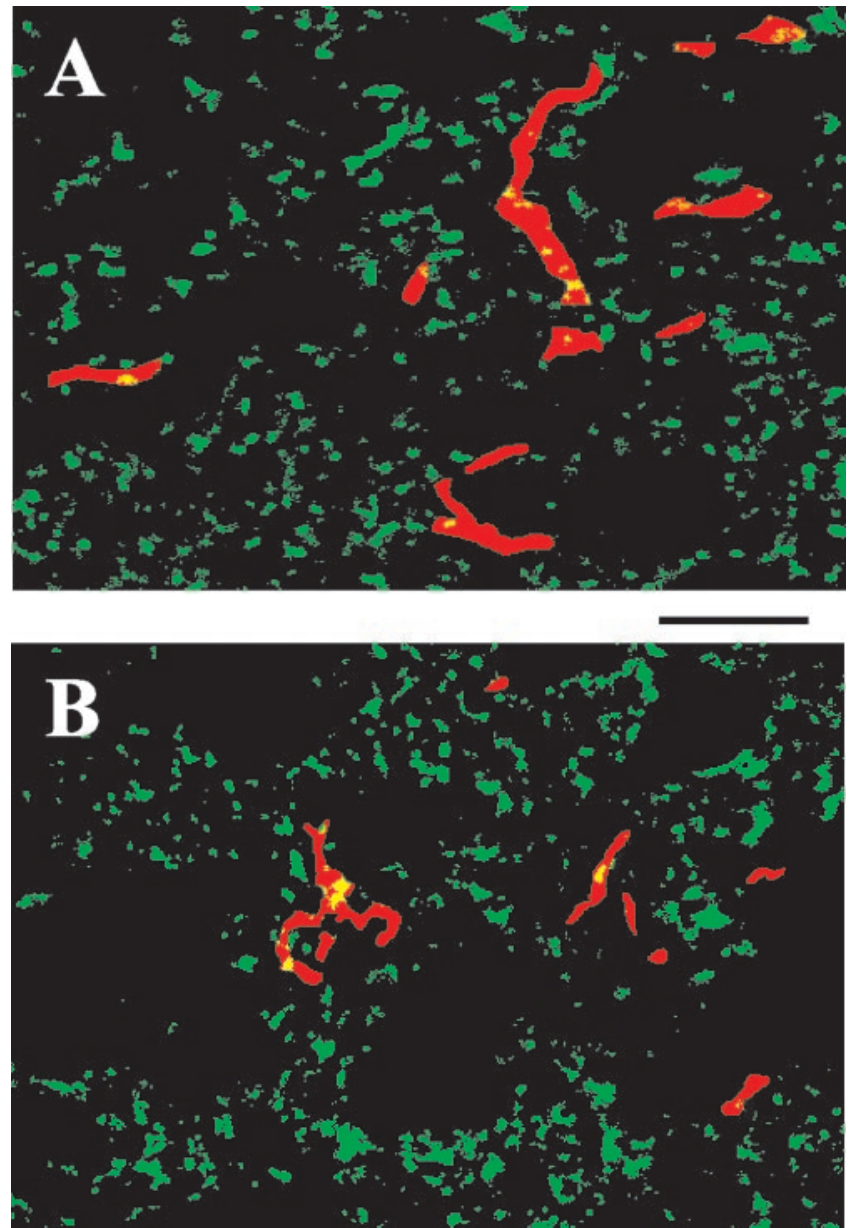

Figure 7. Double-label immunofluorescent confocal images from the border $(A)$ and center $(B)$ of the scotoma in an animal injected with BDNF into the eye and an animal infused with $C-A B C$, respectively. In both $A$ and $B$, the green image represents a single confocal section in which the brightest $10 \%$ of the synapsin I-positive pixels in the neuropil have been highlighted. The CTB-labeled axon branches that are located in the focal plane corresponding to synapsin immunolabeling are highlighted in red. Synaptic puncta colocalizing with retinal sprouts (yellow) are evident in both images. Scale bar, $10 \mu \mathrm{m}$.

throughout the scotoma, thus confirming the robust effects of the combined treatment on fiber sprouting (Fig. 6E). As an additional control, we measured the intensity of retinal fiber labeling in uninjured areas of the SC in control and treated animals. We found that CTB labeling was not significantly different either among individual animals (one-way ANOVA, $p=0.11$ ) or between the two groups ( $t$ test, $p=0.23$ ).

\section{Synapse formation by the sprouting axons}

A very important issue is whether retinal fibers sprouting into the collicular scotoma are able to establish synapses with the deafferented tectal cells. To address this issue, we performed doublelabel immunofluorescence for CTB and the synaptic vesicle marker synapsin I. Confocal images of sections stained for synapsin I showed brightly immunoreactive puncta in the neuropil, likely corresponding to individual presynaptic terminals, and absence of label in cell bodies (Fig. 7). A conservative, object-based colocalization analysis was performed to assess the presence of synaptic puncta within labeled retinal fibers using the methods and the software described by Silver and Stryker (2000a). This analysis demonstrated the colocalization of synapsin immunostaining and RGC fibers at the border of the collicular scotoma in 
BDNF-injected animals (Fig. 7A). Remarkably, synaptic terminals colocalized with retinal axons also in the center of the scotoma of animals treated with C-ABC (Fig. $7 B$ ). These results indicate that retinal fibers invading the denervated tectum establish connections with the postsynaptic targets.

\section{Analysis of synaptic density}

It has been previously shown by electron microscopy that removal of the retinal input in the rat results in a reduction of synaptic density in the deafferented SC (Lund and Lund, 1971). Prompted by the effects of BDNF and C-ABC on retinal fiber sprouting and synaptogenesis, we checked whether total synaptic density within the scotoma is also affected by these treatments. We have addressed this issue using confocal laser-scanning microscopy to quantify immunohistochemical staining for synapsin I. We measured the area occupied by the immunoreactive staining in fields taken at the border and in the center of the collicular scotoma. These values were normalized to the data obtained in the adjacent undeprived superficial gray of the same SC (see Materials and Methods). We found that in untreated lesioned rats at 1 week, synaptic density was $\sim 65 \%$ of the control value both at the border and in the center of the scotoma, with no significant difference between the two regions ( $t$ test, $p=0.72$ ). We then checked whether any spontaneous reinvasion of synaptic sites occurs over time by analyzing animals taken 3 weeks after surgery. No differences in synaptic density could be detected between animals taken 7 and $21 \mathrm{~d}$ after the lesion, either at the border or in the center of the collicular scotoma ( $t$ test, $p=0.41$; $p=0.62$, respectively; Fig. $8 A, B$ ). Thus, no occupation of synaptic sites occurs spontaneously between 1 and 3 weeks after deafferentation.

We then assessed the effects of BDNF and C-ABC on total synaptic density after 1 week of treatment. At the border of the scotoma, minipump delivery of BDNF was effective in restoring a normal cross-sectional area of synapsin immunolabeling (mean \pm SE, $111 \pm 12 \%$ of the values observed in the adjacent, undeprived superficial gray). The statistical analysis indicates that synaptic density in BDNF-infused rats is significantly increased with respect to that measured in both control lesioned and cytochrome $c$-infused animals (one-way ANOVA, $p<0.05$; post hoc Tukey test, $p<0.05$; Fig. $8 C$ ). Similar results on synaptic density in the border region were obtained by injecting BDNF intraocularly (ANOVA, $p<0.01$; post hoc Tukey test, intravitreal BDNF vs intravitreal cytochrome $c$ and control lesioned rats, $p<$ 0.05; Fig. $8 D$ ). In the center of the scotoma, both $\mathrm{BDNF}$ regimens failed to reach statistical significance (ANOVA, both $p=0.10$; data not shown).

Treatment with C-ABC increased synaptic density at the border of the scotoma, but this effect only approached statistical significance (ANOVA, $p=0.07$; Fig. $8 E$ ). C-ABC was completely ineffective in the center (ANOVA, $p=0.67$ ). Animals that received both BDNF into the eye and $\mathrm{C}-\mathrm{ABC}$ into the $\mathrm{SC}$ showed a restoration of normal synaptic density values (mean \pm SE, $98 \pm$ $7 \%$ of the values obtained in the undeprived superficial gray) at the border of the scotoma ( $t$ test, BDNF plus C-ABC vs untreated animals, $p=0.01$; Fig. $8 F)$, but no significant effect was detected in the center ( $t$ test, $p=0.29$ ). The effects of the combined treatment were superimposable on those of intraocular BDNF alone $(t$ test, BDNF eye vs BDNF plus C-ABC, $p=0.32$ at the border; $p=$ 0.3 in the center).
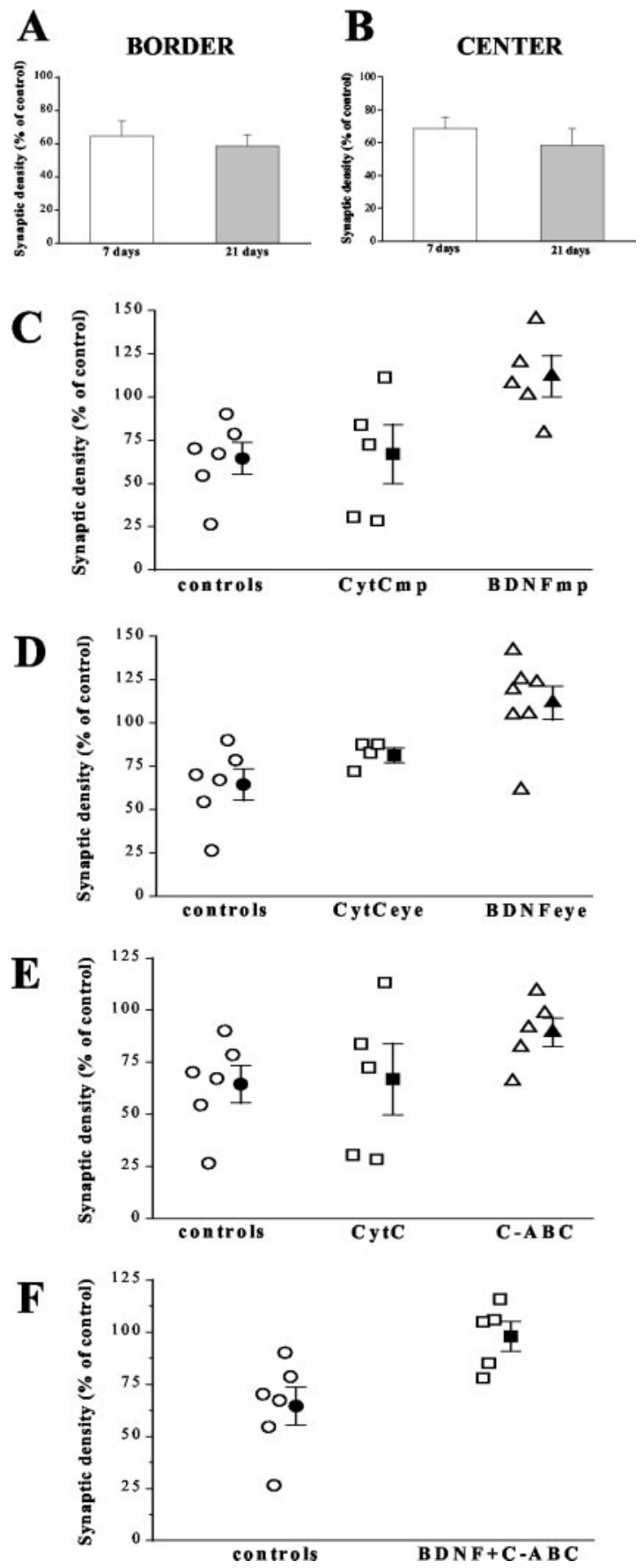

Figure 8. $\quad A, B$, Synaptic density values in untreated animals killed 7 and $21 \mathrm{~d}$ after the lesion. Neither at the border $(A)$ nor in the center of the collicular scotoma $(B)$ are there significant differences between the two time points. Error bars indicate SE. For each bar, $n=6-8$ rats. $C-F$, Effects of the various manipulations on synaptic density at the border of the scotoma. In each panel, controls are untreated lesioned animals. C, Effects of BDNF infused into the SC via osmotic minipumps (BDNFmp) compared with those obtained in animals that received cytochrome c (CytCmp). D, Effects of the intravitreal injection of BDNF (BDNFeye) and cytochrome $c$ as a control (CytCeye). E, C-ABC alone increases synaptic density, but this effect is not significant. $F$, Delivery of BDNF plus C-ABC restores normal synaptic density at the border of the scotoma.

\section{Microglial activation within the collicular scotoma}

Experimental modulation of the inflammatory response has been reported to affect fiber regrowth and plasticity after CNS lesions (Leon et al., 2000). We therefore controlled the inflammatory response in the different treatment groups using staining for OX42 , an antibody that reveals microglia and neutrophils. In control 


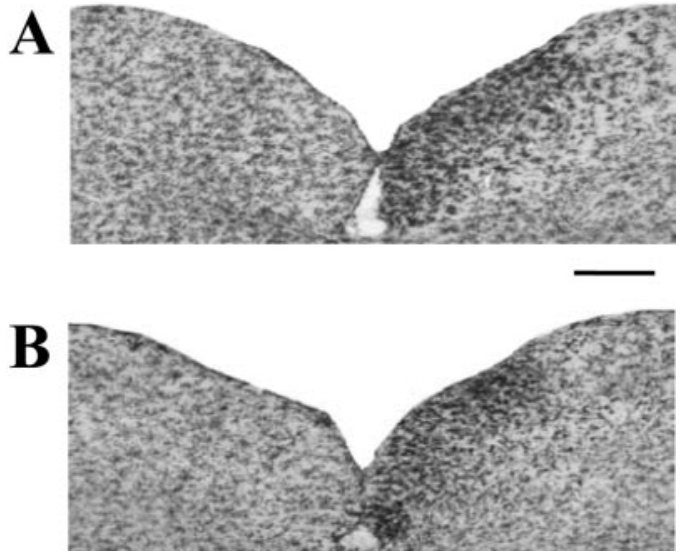

Figure 9. Example of $0 X-42$ immunoreactivity in control lesioned animals $(A)$ and rats treated with BDNF and $C-A B C(B)$. A spot of increased $0 X-42$ staining is visible on the medial part of the SC contralateral to the lesion in both images. Scale bar, $400 \mu \mathrm{m}$.

lesioned animals we observed a strong increase in OX-42 staining that precisely matched the extent of the collicular scotoma, as determined by anterograde labeling with CTB. No qualitative differences could be detected among the groups in both the pattern and intensity of the microglial reactivity (Fig. 9).

\section{Discussion}

In the present paper we have identified experimental treatments that allow retinal fiber sprouting and synaptogenesis within the collicular scotoma after a partial retinal lesion in the adult rat. We report that the combined delivery of $\mathrm{BDNF}$ and $\mathrm{C}-\mathrm{ABC}$ allows the reinnervation of the collicular scotoma by unlesioned RGC axons.

\section{Methodological considerations}

Many studies have shown that the return of function after CNS injury can be mediated by plastic sprouting of the undamaged fiber tracts (Thallmair et al., 1998; Chen et al., 2002a,b). It is fundamental, therefore, to identify experimental repair strategies that stimulate unlesioned axons to grow collaterals and to form new synaptic contacts. To assess quantitatively the impact of any experimental treatment on fiber sprouting and synaptogenesis, one needs a model of a CNS lesion in which these measures can be accurately performed. Our model of a partial retinal lesion is particularly suited to this aim for several reasons. First, the precise topographical organization of the retinotectal projection allows one to produce via focal retinal lesions a zone of target denervation of extremely reproducible size and location. Second, by depriving of the retinal input only a small part of the contralateral SC, we were able to give a quantitative evaluation of both fiber sprouting and synaptogenesis. Fiber density and synaptic density within the scotoma were normalized to those present in the adjacent, undeprived SC of each coronal section, thus compensating for possible changes in the anterograde labeling efficiency and in the quality of the immunostaining between individual sections and animals. Third, the use of a highly sensitive anterograde tracer such as CTB in conjunction with confocal microscopy permits visualization of single axon collaterals that have sprouted into the collicular scotoma.

\section{Absence of spontaneous plasticity after deafferentation \\ As a first experimental aim, we were interested in assessing} whether some axonal plasticity occurs spontaneously over time.
Our quantitative analysis did not detect any significant differences in the sprouting response between 1 and 3 weeks after the lesion. Similarly, corticocollicular fibers are not able to occupy denervated territories of the SC after enucleation in the adult rat (Garcia del Cano et al., 2002). We have also determined whether a reoccupation of synaptic sites occurs spontaneously after deafferentation. To address this issue, we measured the crosssectional area of synapsin immunoreactivity as an indicator of the overall density of presynaptic boutons (Smith et al., 2000). Immunolabeling for synaptic vesicle proteins has been shown to provide a sensitive and reliable method for determining synapse density, and results from this approach are in close agreement with those obtained by electron microscopy (Silver and Stryker, 2000b; Smith et al., 2000). Quantification of synapsin staining indicates that $\sim 35 \%$ fewer synapses are present within the scotoma at 1 week, and this value is unaltered at 3 weeks. Thus, the adult tectum appears to be particularly resistant to attempts at filling in by unlesioned axons. This could potentially be caused by intrinsic factors, i.e., a complete inability of the adult RGCs to extend axon collaterals, or by extrinsic ones, i.e., the presence of neurite growth inhibitors in the adult SC. We attempted to increase the sprouting potential of the unlesioned RGCs via BDNF treatment and to neutralize repulsive factors through the use of C-ABC.

\section{Effects of BDNF}

We have used the administration of BDNF to enhance the intrinsic potential of RGCs to grow collaterals and to establish new synaptic connections. The choice of BDNF stems from several pieces of evidence recently reported in the literature. First, in vitro data indicate that BDNF is the most powerful factor for stimulating RGC axon growth (Goldberg et al., 2002). These experiments, conducted on cultures of highly purified RGCs, allow the conclusion that BDNF signals axon elongation by modifying the intrinsic growth state of RGCs independent of extracellular influences (Goldberg et al., 2002). Second, RGCs express high levels of the high-affinity BDNF receptor TrkB (Cellerino and Kohler, 1997) and respond to BDNF stimulation in vivo with a prominent upregulation of several growth-associated genes (Klocker et al., 2001). Finally, BDNF enhances synaptogenesis of RGC axons in vivo (Alsina et al., 2001). Here we have found that BDNF treatment is very effective in increasing RGC fiber sprouting and synaptic density at the border of the collicular scotoma after 1 week of treatment. These data demonstrate for the first time an effect of BDNF on sprouting of unlesioned axons and synapse formation after deafferentation in the CNS. Interestingly, the effects produced by the intraocular administration of BDNF were undistinguishable from those observed after BDNF release into the SC. This could be explained by taking into account the finding that exogenous BDNF is transported both anterogradely and retrogradely along the optic nerve (von Bartheld, 1998; Caleo et al., 2000), so that delivery of BDNF at either location will result in the activation of BDNF-related signal transduction cascades both at the RGC bodies and in the tectum. However, recent data show that the location at which a neurotrophin stimulates a cell is a crucial parameter in determining the signaling pathways that are activated and the ensuing biological responses (Watson et al., 2001; Lom et al., 2002) (for review, see Heerssen and Segal, 2002). Therefore, the comparable results obtained with different routes of BDNF administration in the present study must be ascribed to the fact that distinct BDNF-mediated signal transduction pathways converge on common effectors mediating the reparative response. 
Despite promoting extensive collateralization at the border, BDNF treatment failed to elicit similar results in the center of the scotoma. This result is consistent with the observation that neurotrophins are not able to counteract the nonpermissive nature of the CNS (Horner and Gage, 2000; Markus et al., 2002). Thus, enhancing the growth potential of RGCs with BDNF stimulation is not sufficient to induce long-range neurite extension, possibly because of the contrasting action of neurite growth inhibitors in the tissue parenchyma (Horner and Gage, 2000).

\section{Effects of C-ABC}

To remove extracellular inhibitory influences, we have exploited the bacterial enzyme C-ABC, which degrades CSPGs. CSPGs are components of the extracellular matrix that are made up of a protein core equipped with many side chains, known as GAG chains, and consisting of 20-200 repeated disaccharide units (Bandtlow and Zimmermann, 2000). Much of the inhibitory activity of CSPGs toward axon growth can be removed by degradation of glycosaminoglycan chains with C-ABC (McKeon et al., 1995; Smith-Thomas et al., 1995; Zuo et al., 1998). Indeed, it has been shown that $\mathrm{C}-\mathrm{ABC}$ treatment promotes regeneration of severed fibers in the nigrostriatal tract (Moon et al., 2001). Recent data demonstrate that $\mathrm{C}-\mathrm{ABC}$ promotes regeneration and functional recovery after spinal cord injury (Bradbury et al., 2002). In addition, administration of $\mathrm{C}$ - $\mathrm{ABC}$ restores plasticity to the adult visual cortex (Pizzorusso et al., 2002). However, there is no direct evidence supporting an involvement of CSPGs in the control of the sprouting of unlesioned central tracts. Our data show that $\mathrm{C}-\mathrm{ABC}$ injections into the denervated SC are effective in increasing RGC fiber sprouting not only at the border but also in the center of the collicular scotoma. These results support the hypothesis that long-range RGC axon growth critically depends on inhibitory molecules such as CSPGs that are present in the SC parenchyma. The data also demonstrate for the first time a crucial role for CSPGs in restricting plasticity of adult fiber tracts after deafferentation.

In $\mathrm{C}$-ABC-infused rats we have also shown that the newly formed RGC collaterals make synapses with tectal cells in the center of the scotoma. These findings suggest that the C-ABCmediated induction of retinal sprouts may render the collicular scotoma directly responsive to the afferent retinal input. Our data are also consistent with previous electrophysiological data showing that $\mathrm{C}-\mathrm{ABC}$ treatment promotes formation of functional connections by regenerated axons (Bradbury et al., 2002). When total synaptic density was assessed, however, C-ABC failed to induce significant effects within the scotoma. An increase of RGC synapses after $\mathrm{C}$-ABC delivery could have escaped detection by our protocol, because a modest increase in the number of RGC synapses affects total synaptic density only marginally.

\section{Effects of the combined treatment with BDNF and C-ABC}

A crucial question that we have addressed in the present study is whether the plastic reorganization of RGC fibers can be boosted by simultaneously increasing the growth potential of RGCs and reducing extracellular inhibitory influences via CSPG degradation. To this aim, we combined delivery of BDNF into the eye and injections of $\mathrm{C}-\mathrm{ABC}$ into the SC. This protocol proved to be dramatically more effective than either treatment alone in promoting fiber sprouting at the border of the scotoma (see Fig. 5). Indeed, the combination of BDNF and C-ABC showed synergistic effects, indicating that formation of collaterals is powerfully stimulated under conditions in which RGC fibers are simultaneously made "growth-enabled" by BDNF and face a more per- missive environment. Therefore, the two agents potentiate each other in the control of beneficial compensatory sprouting mechanisms.

A possible explanation for the synergistic action of BDNF and $\mathrm{C}-\mathrm{ABC}$ is that the C-ABC-mediated degradation of CSPGs increases the availability of BDNF via enhanced diffusion of the neurotrophin in the tissue parenchyma. To address this issue, we performed Western blot analysis of Trk receptor phosphorylation on protein extracts prepared from the SC of either normal rats or rats that were injected into the $\mathrm{SC}$ with $\mathrm{C}-\mathrm{ABC}$ or a control solution. We measured the relative amount of phosphorylated Trk with respect to the total amount of Trk present in the SC. This ratio was $0.43 \pm 0.09$ (mean $\pm \mathrm{SE}$ ) in normal rats, $0.32 \pm 0.13$ in animals injected with $\mathrm{C}-\mathrm{ABC}$, and $0.47 \pm 0.07$ in animals injected with the control solution. ANOVA did not show significant differences among the experimental groups $(p=0.38)$. These results suggest that $\mathrm{C}-\mathrm{ABC}$ acts independently from $\mathrm{BDNF}$, although we cannot rule out that BDNF signaling downstream of Trk activation is indirectly influenced by C-ABC.

Whatever the mechanisms involved in the synergism, our findings indicate that the combination of a neurotrophic factor, which activates cellular responses needed for neurite growth, with agents that change the brain parenchyma into a more permissive environment is particularly effective in triggering processes of plastic reorganization that are fundamental for the return of function after CNS injury. If extended to other fiber systems in the brain, these findings may be relevant in therapies for the repair of the damaged adult CNS.

\section{References}

Alsina B, Vu T, Cohen-Cory S (2001) Visualizing synapse formation in arborizing optic axons in vivo: dynamics and modulation by BDNF. Nat Neurosci 4:1093-1101.

Bandtlow CE, Zimmermann DR (2000) Proteoglycans in the developing brain: new conceptual insights for old proteins. Physiol Rev 80:1267-1290.

Bastos EF, Marcelino JL, Amaral AR, Serfaty CA (1999) Fluoxetine-induced plasticity in the rodent visual system. Brain Res 824:28-35.

Berry M, Carlile J, Hunter A (1996) Peripheral nerve explants grafted into the vitreous body of the eye promote the regeneration of retinal ganglion cell axons severed in the optic nerve. J Neurocytol 25:147-170.

Bradbury EJ, Moon LD, Popat RJ, King VR, Bennett GS, Patel PN, Fawcett JW, McMahon SB (2002) Chondroitinase ABC promotes functional recovery after spinal cord injury. Nature 416:636-640.

Brittis PA, Flanagan JG (2001) Nogo domains and a Nogo receptor: implications for axon regeneration. Neuron 30:11-14.

Caleo M, Menna E, Chierzi S, Cenni MC, Maffei L (2000) Brain-derived neurotrophic factor is an anterograde survival factor in the rat visual system. Curr Biol 10:1155-1161.

Cellerino A, Kohler K (1997) Brain-derived neurotrophic factor/neurotrophin-4 receptor TrkB is localized on ganglion cells and dopaminergic amacrine cells in the vertebrate retina. J Comp Neurol 386:149-160.

Chen P, Goldberg DE, Kolb B, Lanser M, Benowitz LI (2002a) Inosine induces axonal rewiring and improves behavioral outcome after stroke. Proc Natl Acad Sci USA 99:9031-9036.

Chen R, Cohen LG, Hallett M (2002b) Nervous system reorganization following injury. Neuroscience 111:761-773.

Chierzi S, Fawcett JW (2001) Regeneration in the mammalian optic nerve. Restor Neurol Neurosci 19:109-118.

Cohen-Cory S, Fraser SE (1995) Effects of brain-derived neurotrophic factor on optic axon branching and remodelling in vivo. Nature 378:192-196.

Colonnese MT, Constantine-Paton M (2001) Chronic NMDA receptor blockade from birth increases the sprouting capacity of ipsilateral retinocollicular axons without disrupting their early segregation. J Neurosci 21:1557-1568.

Domeniconi M, Cao Z, Spencer T, Sivasankaran R, Wang K, Nikulina E, Kimura N, Cai H, Deng K, Gao Y, He Z, Filbin M (2002) Myelinassociated glycoprotein interacts with the Nogo66 receptor to inhibit neurite outgrowth. Neuron 35:283-290. 
Fouad K, Dietz V, Schwab ME (2001) Improving axonal growth and functional recovery after experimental spinal cord injury by neutralizing myelin associated inhibitors. Brain Res Rev 36:204-212.

Fournier AE, Strittmatter SM (2001) Repulsive factors and axon regeneration in the CNS. Curr Opin Neurobiol 11:89-94.

Garcia del Cano G, Gerrikagoitia I, Martinez-Millan L (2002) Plastic reaction of the rat visual corticocollicular connection after contralateral retinal deafferentiation at the neonatal or adult stage: axonal growth versus reactive synaptogenesis. J Comp Neurol 446:166-178.

Goldberg JL, Espinosa JS, Xu Y, Davidson N, Kovacs GT, Barres BA (2002) Retinal ganglion cells do not extend axons by default: promotion by neurotrophic signaling and electrical activity. Neuron 33:689-702.

Heerssen HM, Segal RA (2002) Location, location, location: a spatial view of neurotrophin signal transduction. Trends Neurosci 25:160-165.

Horner PJ, Gage FH (2000) Regenerating the damaged central nervous system. Nature 407:963-970.

Klocker N, Jung M, Stuermer CA, Bahr M (2001) BDNF increases the number of axotomized rat retinal ganglion cells expressing GAP-43, L1, and TAG-1 mRNA: a supportive role for nitric oxide? Neurobiol Dis 8:103-113.

Lehmann M, Fournier A, Selles-Navarro I, Dergham P, Sebok A, Leclerc N, Tigyi G, McKerracher L (1999) Inactivation of Rho signaling pathway promotes CNS axon regeneration. J Neurosci 19:7537-7547.

Leon S, Yin Y, Nguyen J, Irwin N, Benowitz LI (2000) Lens injury stimulates axon regeneration in the mature rat optic nerve. J Neurosci 20:4615-4626.

Lom B, Cogen J, Sanchez AL, Vu T, Cohen-Cory S (2002) Local and targetderived brain-derived neurotrophic factor exert opposing effects on the dendritic arborization of retinal ganglion cells in vivo. J Neurosci 22:7639-7649.

Lund RD, Lund JS (1971) Synaptic adjustment after deafferentation of the superior colliculus of the rat. Science 171:804-807.

Lund RD, Cunningham TJ, Lund JS (1973) Modified optic projections after unilateral eye removal in young rats. Brain Behav Evol 8:51-72.

Markus A, Patel T, Snider W (2002) Neurotrophic factors and axonal growth. Curr Opin Neurobiol 12:523-531.

McKeon RJ, Hoke A, Silver J (1995) Injury-induced proteoglycans inhibit the potential for laminin-mediated axon growth on astrocytic scars. Exp Neurol 136:32-43.

Moon LD, Asher RA, Rhodes KE, Fawcett JW (2001) Regeneration of CNS axons back to their target following treatment of adult rat brain with chondroitinase ABC. Nat Neurosci 4:465-466.

Pizzorusso T, Medini P, Berardi N, Chierzi S, Fawcett JW, Maffei L (2002)
Reactivation of ocular dominance plasticity in the adult visual cortex. Science 298 (5596):1187-1189.

Raineteau O, Schwab ME (2001) Plasticity of motor systems after incomplete spinal cord injury. Nat Rev Neurosci 2:263-273.

Silver MA, Stryker MP (2000a) A method for measuring colocalization of presynaptic markers with anatomically labeled axons using double label immunofluorescence and confocal microscopy. J Neurosci Methods 94:205-215.

Silver MA, Stryker MP (2000b) Distributions of synaptic vesicle proteins and GAD65 in deprived and nondeprived ocular dominance columns in layer IV of kitten primary visual cortex are unaffected by monocular deprivation. J Comp Neurol 422:652-664.

Smith TD, Adams MM, Gallagher M, Morrison JH, Rapp PR (2000) Circuit-specific alterations in hippocampal synaptophysin immunoreactivity predict spatial learning impairment in aged rats. J Neurosci 20:6587-6593.

Smith-Thomas LC, Stevens J, Fok-Seang J, Faissner A, Rogers JH, Fawcett JW (1995) Increased axon regeneration in astrocytes grown in the presence of proteoglycan synthesis inhibitors. J Cell Sci 108:1307-1315.

So KF, Yip HK (1998) Regenerative capacity of retinal ganglion cells in mammals. Vision Res 38:1525-1535.

Thallmair M, Metz GA, Z'Graggen WJ, Raineteau O, Kartje GL, Schwab ME (1998) Neurite growth inhibitors restrict plasticity and functional recovery following corticospinal tract lesions. Nat Neurosci 1:124-131.

Viegi A, Cotrufo T, Berardi N, Mascia L, Maffei L (2002) Effects of dark rearing on phosphorylation of neurotrophin Trk receptors. Eur J Neurosci 16:1925-1930.

von Bartheld CS (1998) Neurotrophins in the developing and regenerating visual system. Histol Histopathol 13:437-459.

Watson FL, Heerssen HM, Bhattacharyya A, Klesse L, Lin MZ, Segal RA (2001) Neurotrophins use the Erk5 pathway to mediate a retrograde survival response. Nat Neurosci 4:981-988.

Weidner N, Ner A, Salimi N, Tuszynski MH (2001) Spontaneous corticospinal axonal plasticity and functional recovery after adult central nervous system injury. Proc Natl Acad Sci USA 98:3513-3518.

Z'Graggen WJ, Metz GA, Kartje GL, Thallmair M, Schwab ME (1998) Functional recovery and enhanced corticofugal plasticity after unilateral pyramidal tract lesion and blockade of myelin-associated neurite growth inhibitors in adult rats. J Neurosci 18:4744-4757.

Zuo J, Neubauer D, Dyess K, Ferguson TA, Muir D (1998) Degradation of chondroitin sulfate proteoglycan enhances the neurite-promoting potential of spinal cord tissue. Exp Neurol 154:654-662. 